\title{
CHAOTIC BEHAVIOR IN PIECEWISE CONTINUOUS DIFFERENCE EQUATIONS
}

BY

JAMES P. KEENER ${ }^{1}$

\begin{abstract}
A class of piecewise continuous mappings with positive slope, mapping the unit interval into itself is studied. Families of 1-1 mappings depending on some parameter have periodic orbits for most parameter values, but have an infinite invariant set which is a Cantor set for a Cantor set of parameter values. Mappings which are not 1-1 exhibit chaotic behavior in that the asymptotic behavior as measured by the rotation number covers an interval of values. The asymptotic behavior depends sensitively on initial data in that the rotation number is either a nowhere continuous function of initial data, or else it is a constant on all but a Cantor set of the unit interval.
\end{abstract}

1. Introduction. In this paper we discuss the solutions of the difference equation

$$
x_{n+1}=F\left(x_{n}\right)
$$

where $F$ is a piecewise continuous function which maps some interval $I$ into itself. It is well known [1], [2] that if $F$ is continuous on $I$ and (1.1) possesses a solution of period 3, that is, if there is a point $x \in I$ such that $x=F^{(3)}(x)=F(F(F(x)))$ and $x \neq F(x)$, then (1.1) possesses solutions of all periods as well as many aperiodic solutions, depending sensitively on the initial point $x_{0}$. The requirement that $F$ must be a continuous function is a restriction that may not be reasonable in some applications even though the solutions of (1.1) may be "chaotic".

There are many ways that discontinuities can influence the shape of a function $F$. In this paper we restrict our attention to functions $F$ which have a single jump discontinuity and which are continuous and (locally) increasing elsewhere. This class of functions occurs in the study of periodic stimulation of excitable media [3], [4], [5]. Other physical situations may suggest other important classes of functions, but we have chosen to overlook them here.

Suppose $F$ has a jump discontinuity at $x=\theta$. At $x=\theta, F$ has left- and right-hand limits denoted

$$
b=\lim _{x \uparrow \theta} F(x), \quad a=\lim _{x \downarrow \theta} F(x) .
$$

If $a>b$ or if $a<b$, but $a>\theta$ or $b<\theta$, the discontinuity at $\theta$ has little effect on the iterates of $F$. The only case with sufficient complexity to warrant in-depth study is $a<\theta<b$. All of our attention will be focused on the interval $[a, b]$ and we will

Received by the editors October 2, 1978.

AMS (MOS) subject classifications (1970). Primary 58F20.

Key words and phrases. Chaotic behavior, rotation number, circle mappings.

${ }^{1}$ This research was supported in part by the National Science Foundation under Grant MPS 75-07621.

(C) 1980 American Mathematical Society 0002-9947/80/0000-0462/\$05.00 
assume that $F$ maps $[a, b]$ into itself. The mapping need not be onto or $1-1$, but in order for $[a, b]$ to map into itself we require

$$
F(a) \geqslant a, \quad F(b) \leqslant b .
$$

By a straightforward change of variables, the mapping $F$ can be transformed into a mapping of the unit interval $[0,1]$ into itself. Define

$$
\tilde{F}(x)=\frac{F((b-a) x+a)-a}{b-a}
$$

and notice that $\tilde{F}$ maps $[0,1]$ into $[0,1]$, has a jump discontinuity at $\tilde{\theta}=$ $(\theta-a) /(b-a)$ and is increasing elsewhere. Without loss of generality, then, we will regard $F$ as one such function on $[0,1]$. A typical plot of the function $F$ is shown in Figure 1. We assume that $F$ satisfies the properties

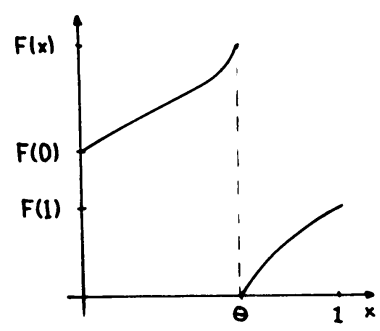

FIGURE 1

F-I. $F(x)$ is a piecewise continuously differentiable function defined on the interval $[0,1]$ with a single jump discontinuity at $x=\theta \in(0,1)$.

F-II. For $x \neq \theta, 0<t \leqslant d F / d x \leqslant s<\infty$.

F-III. $\lim _{x \downarrow \theta} F(x)=0, \lim _{x \uparrow \theta} F(x)=1$ and $F(\theta)=0$ or 1 .

It is convenient to view the mapping $F$ as a mapping of a circle into itself. If we identify 0 with 1 as a point on a circle, then $F$ can be visualized as follows. Cut the circle at the point zero, stretch, shrink, or rotate the cut line segment and lay it back onto the circle. In this interpretation the point $\theta$ is not a discontinuity at all, but is simply the image of a point which is mapped into the origin to be "cut" on the next iterate. The true discontinuity of this map is at the zero of the circle since $F(0) \neq F(1)$ in general.

As we shall see, $F(0)>F(1)$ leads to results vastly different from $F(0)<F(1)$. These cases will be loosely referred to as the nonoverlapping and overlapping cases, respectively, because of their interpretation on the circle. In the first case $F$ maps $[0,1]$ into but not onto itself and in the second case $F$ maps $[0,1]$ onto itself but is not $1-1$. The dividing case $F(0)=F(1)$ is exactly the case of a continuous monotone map of a circle onto itself studied extensively by Denjoy and others [6], [7], [8].

The main reason to view $F$ as a map of the circle is because of the useful concept of rotation number.

DefinITION 1.1. For each point $x \in[0,1]$ define the rotation number $\rho(x)$ to be

$$
\rho(x)=\lim _{n \rightarrow \infty} \frac{1}{n} \sum_{k=1}^{n} X_{\theta}\left(F^{n}(x)\right)
$$

where $X_{\theta}$ is the characteristic function for the interval $(\theta, 1]$. 
Although this is not the way the rotation number is usually defined [7] it is equivalent to other definitions. The rotation number as defined here counts the number of times the iterates of a point $x$ are on the right $\theta$, signifying a complete rotation of a circle. For example, if $\rho(x)=p / q, p, q$ integers, then the images of the point $x$ make exactly $p$ rotations of the circle in $q$ iterates.

According to the title of this paper, we intend to study the chaotic behavior of the mapping $F$. Exactly what is meant by chaos is in itself a confusing question. One definition of chaos, taken from $\mathrm{Li}$ and Yorke [2], is that the mapping (1.1) has periodic solutions of all possible periods and many aperiodic solutions as well. A second description of chaos is that the mapping $F$ is equivalent to a shift on a sequence space [8], [9]. This definition is slightly more general than the first in that the sequence space may be restricted to eliminate solutions of certain periods.

The definition of chaos which we will use is as follows.

Definition 1.2. The motion of (1.1) is said to be chaotic if the range of the rotation number $\rho(x)$ covers some nonzero interval $[\alpha, \beta], \alpha \neq \beta$ for $x \in[0,1]$.

According to this definition the long time behavior of an iterate depends sensitively on initial data [11], there are many periodic solutions (correspondng to rational rotation number) as well as aperiodic solutions (irrational rotation number). This definition of chaos is slightly more general than the previous two, although it suffers from the fact that not all classes of maps can easily be assigned a natural rotation number.

We can summarize our results as follows.

(i) If $F(0)>F(1)$ (nonoverlapping) the rotation number $\rho(x)$ is a constant independent of initial data $x$. If $F$ is a member of a family of such maps depending smoothly on some parameter $\lambda$, then $\rho(x ; \lambda)$ is a Cantor function of $\lambda$ which assumes the rational values on nonempty intervals and the irrational values on a Cantor set in $\lambda$ (uncountable, measure zero). When $\rho$ is rational, (1.1) has periodic orbits, and when $\rho$ is irrational the invariant set of $F, \cap_{k=0}^{\infty} F^{(k)}([0,1])$, is a Cantor set.

(ii) If $F(0)<F(1)$ (overlapping) the rotation number $\rho(x)$ covers a nontrivial interval for $x \in[0,1]$ provided $F(1)-F(0)$ is sufficiently large. If $t>1$ (see F-II) then $\rho(x)$ is a nowhere continuous function of $x$. If $t<1$ then $\rho(x)$ may be constant on some interval, in which case it takes on other values on at most a Cantor set (which may have positive measure). Roughly speaking, the range of the function $\rho(x)$ increases with the amount of overlap $F(1)-F(0)$.

In the sections that follow we will discuss these results and some consequences in more detail. In $\$ 2$ we give some of the technical preliminaries necessary for the proofs that follow. $\$ 3$ deals with strictly into (nonoverlapping) maps and $\$ 4$ with noninvertible (overlapping) maps.

2. Technical preliminaries. Probably the easiest way to demonstrate chaos for a mapping such as (1.1) is to show that (1.1) is equivalent to a shift on a sequence space. Since this approach to finding chaotic behavior will be useful to us in later sections we will outline in a series of lemmas the ingredients needed for this type of "chaos" to occur. The lemmas we present are adaptations of those used in [2] to 
take into account the possibility of (many) jump discontinuities.

Lemma 2.1. Suppose $F$ is continuous on the closed interval $I_{0}$. For any closed interval $I_{1} \subset F\left(I_{0}\right)$ there is a closed interval $Q_{0} \subset I_{0}$ such that $F\left(Q_{0}\right)=I_{1}$.

Proof. Let $I_{1}=[F(p), F(q)]$ where $p, q \in I_{0}$. If $p<q$, let $r$ be the last point of $[p, q]$ where $F(r)=F(p)$ and let $s$ be the first point after $r$ where $F(s)=F(q)$. Then $F([r, s])=I_{1}$ and $Q_{0}=[r, s]$.

LEMMA 2.2. Suppose $F$ maps the interval I into itself. Let $\left\{I_{n}\right\}$ be a sequence of closed subintervals $I_{n} \subset I$ with $F$ continuous on $\cup_{n} I_{n}$ and $I_{n+1} \subset F\left(I_{n}\right)$ for all $n$. Then there is a sequence of closed intervals $Q_{n}$ such that $Q_{n+1} \subset Q_{n} \subset I_{0}$ and $F^{(n)}\left(Q_{n}\right)=I_{n}$ for $n \geqslant 0$. For any $x \in Q=\cap_{n} Q_{n}, F^{(n)}(x) \in I_{n}$ for all $n$.

Proof. Notationally, by $F^{(n)}(x)$ we mean the $n$th iterate of $x$ defined inductively by $F^{(1)}(x)=F(x)$ and $F^{(n)}(x)=F\left(F^{(n-1)}(x)\right)$. Define $Q_{0}=I_{0}$ so that $F^{(0)}\left(Q_{0}\right)=$ $I_{0}$. If $Q_{n-1}$ is defined so that $F^{(n-1)}\left(Q_{n-1}\right)=I_{n-1}$, then $I_{n} \subset F\left(I_{n-1}\right)=F^{(n)}\left(Q_{n-1}\right)$. By Lemma 2.3 applied to $F^{(n)}$ on $Q_{n-1}$, there is a closed interval $Q_{n} \subset Q_{n-1}$ such that $F^{(n)}\left(Q_{n}\right)=I_{n}$, which completes the induction.

LEMMA 2.3. Suppose the function $g$ is continuous on $[a, b]$ and either

(i) $[a, b] \subset g([a, b])$ or

(ii) $g([a, b]) \subset[a, b]$.

Then $g$ has a fixed point $x \in[a, b], g(x)=x$.

Proof. If $[a, b] \subset g([a, b])$ there are points $\alpha, \beta \in[a, b]$ such that $g(\alpha)=a \leqslant \alpha$, $g(\beta)=b \geqslant \beta$. Thus $g(\alpha)-\alpha \leqslant 0$ and $g(\beta)-\beta \geqslant 0$ so that by continuity $g(x)$ $-x=0$ for some $x \in[\alpha, \beta] \subset[a, b]$. A similar proof works for case (ii).

The way to use these lemmas to exhibit chaos in a specific setting is straightforward. We try to find a set of closed intervals on which $F$ is continuous such that the image of one covers one or more of the intervals. The image of at least one of the intervals must cover at least two such intervals for chaos to result. From the set of inclusions for $F$, a sequence space of symbols (the names of the intervals) is derived and Lemma 2.2 tells us that for every admissible sequence of intervals there is a point in $I$ whose iterates follow the sequences exactly.

There are myriad ways that this approach can yield chaos. To illustrate in specific fashion for the functions of $\$ 1$ we give the following example.

Theorem 2.4. Let $F$ satisfy F-I, II, III and suppose $F(1)<\theta$. Suppose (1.1) has period $p$ and period $q$ solutions, $p \neq q$, and that exactly one point of each of these periodic orbits is greater than $\theta$. Then (1.1) is a shift on a sequence space.

Proof. Let $\left\{y_{i}\right\}_{i=1}^{p}$ and $\left\{z_{i}\right\}_{i=1}^{q}$ be the points of the periodic solution such that $y_{i+1}=F\left(y_{i}\right), y_{i}=F\left(y_{p}\right), y_{p}>\theta$. Therefore $y_{1}<y_{2}<\cdots<y_{p-1}<\theta<y_{p}$ and similarly $z_{1}<z_{2}<\cdots<z_{q-1}<\theta<z_{q}$. Suppose $p<q$. Then $z_{q}<y_{p}$ and

$$
z_{1}<z_{2}<\cdots<z_{k+1}<y_{1}<z_{k+2}<y_{2}<\cdots<z_{q-1}<y_{p-1}<\theta
$$

where $k=q-p$. 
Define the set of intervals $A_{j}=\left[z_{j}, z_{j+1}\right], j=1,2, \ldots, k, B_{j}=\left[z_{k+j}, y_{j}\right], j=$ $1,2, \ldots, p$. It is apparent that $F\left(A_{i}\right) \supset A_{i+1}, i=1,2, \ldots, k-1, F\left(A_{k}\right) \supset B_{1}$, $F\left(B_{i}\right) \supset B_{i+1}, i=1,2, \ldots, p-1$ and $F\left(B_{p}\right) \supset A_{1} \cup A_{2} \cup \cdots \cup A_{k} \cup B_{1}$. Since $k \geqslant 1, F\left(B_{p}\right)$ covers at least two intervals and we have a sequence space of symbols $\left\{A_{1}, A_{2}, \ldots, A_{k}, B_{1}, B_{2}, \ldots, B_{p}\right\}$. An admissible sequence is any sequence made up of strings of the form $\left\{A_{j}, A_{j+1}, \ldots, A_{k}, B_{1}, B_{2}, \ldots, B_{p}\right\}$ where the first element of the string can be any of the $A_{i}$ or $B_{1}$. By Lemma 2.2, for any admissible sequence of intervals there is a point $x \in[0,1]$ such that $F^{(n)}(x)$ follows the given sequence.

This technique is applicable to many classes of functions other than the one studied here. Its application is routine once the general shape of the functions is known, and the image of certain intervals can be deduced. On the other hand this technique gives no measure of how strongly chaotic the motion can be, or how large the largest space of admissible sequences is. For this reason we choose to use rotation number in our definition of chaos.

3. Strictly into mappings. In order for the ideas of the previous section to be useful, the range of $F$ on some interval must be larger than its domain. We intend to show in this section that if the mapping is strictly into, then chaos in the sense defined earlier does not occur. Nonetheless, the invariant set of the mapping may have an exotic structure.

Suppose the function $F$ satisfies properties F-I, II, III and F-IV: $F(0)>F(1)$.

For this class of functions the rotation number $\rho(x)$ exists and is independent of $x$.

LEMMA 3.1. If $F$ satisfies F-I-IV, the rotation number $\rho(x)$ exists independent of $x$, and is rational if and only if some fixed iterate of $F$ has a fixed point.

The proof of a similar lemma for continuous, 1-1 mappings of the circle given in [7] can be adapted to the current case.

To get an idea of what values $\rho$ can take on we must study the mapping $F$ in more detail. Notice first that there are only three possible arrangements that need to be discussed. These are

(1) $F(0)>\theta, F(1)<\theta$,

(2) $F(0)<\theta, F(1)<\theta$,

(3) $F(1)>\theta, F(0)>\theta$.

Each of these situations guarantees that successive iterates never lie to the left of $\theta$ (cases (1), (3)) or to the right of $\theta$ (cases (1), (2)). In other words, although the image of a point may require many iterates to cross $\theta$ in one direction it will cross $\theta$ again in the return direction after exactly one iterate.

We assume that (1.1) has no fixed points. If it does have a fixed point, the rotation number $\rho$ is zero or one. Without loss of generality we will assume that $F(1)<\theta$. Then there are really only two cases, the first of which is disposed of quite easily.

Lemma 3.2. Suppose $F(0)>\theta$ and $F(1)<\theta$. Then $F$ has a period-two solution of (1.1), and rotation number $\rho=1 / 2$. 
Proof. $F$ is continuous on $(0, \theta) \cup(\theta, 1)$ and $F((0, \theta)) \subset(\theta, 1), F((\theta, 1)) \subset$ $(0, \theta)$ so that $F^{(2)}((0, \theta)) \subset(0, \theta)$. In fact $F^{(2)}(0)>0$ and $\lim _{x \uparrow \theta} F^{(2)}(x)=F(1)<\theta$ so according to Lemma 2.3 there is a point, say $\alpha^{*} \in(0, \theta)$, for which $F^{(2)}\left(\alpha^{*}\right)=$ $\alpha^{*}$. The rotation number $\rho$ is easily calculated as the number of iterates greater than $\theta$ per period of the periodic solution.

To discuss the problem when $F(0)<\theta, F(1)<\theta$ is much more complex. We develop some of the necessary tools in the following paragraphs.

Definition 3.3. The set of preimages of $\theta$, denoted $\Omega$, is the set of points $\left\{\phi_{k}\right\}$ for which

(a) $\phi_{k} \in(0, F(0)]$ and

(b) for each $k>0$ there is a unique positive integer $i_{k}$ with $i_{k+1}>i_{k}$ such that

$$
F^{\left(i_{p}-i_{q}\right)}\left(\phi_{p}\right)=\phi_{q}, \quad p>q, \quad F^{\left(i_{p}\right)}\left(\phi_{p}\right)=\theta
$$

Simply speaking $\Omega$ is the set of preimages of $\theta$ which lie in the interval $(0, F(0)]$. Notice that $F$ and all its iterates are continuously differentiable on any subinterval of $(0, F(0)]$ whose intersection with $\Omega$ is empty, since no point of the subinterval is ever mapped into the discontinuity at $\theta$. Furthermore, since $F(x)$ has positive slope, images of the endpoints of the interval have the same ordering as the endpoints themselves.

LEMMA 3.4. $\Omega$ is not empty.

Proof. Let $n \geqslant 0$ be the least positive integer for which $F^{(n)}(0)>\theta$. Clearly $n \geqslant 2$ and $F^{(n-1)}(0)<\theta$. Thus, $F^{(n-1)}$ is continuous on $[0, F(0)]$ and $\theta \in$ $F^{(n-1)}((0, F(0)])$. Therefore, there is a point $\phi_{1} \in(0, F(0)]$ for which $F^{(n-1)}\left(\phi_{1}\right)=\theta$.

Our later discussion will rely heavily on the existence and location of the point $\phi_{1}$ and its associated integer $i_{1}=n-1$.

LEMMA 3.5. If $\theta$ is not a periodic point of (1.1), then the elements of $\Omega$ are distinct.

Proof. Suppose that $\phi_{p}=\phi_{q}$ for $p>q$. Then $F^{\left(i-i_{q}\right)}\left(\phi_{p}\right)=\phi_{q}=\phi_{p}$ so that $\phi_{p}$ is a periodic point of $F$. However, $F^{\left(i_{p}\right)}\left(\phi_{p}\right)=\theta$ implies that $\theta$ is also a periodic point of $F$, contrary to assumption.

If $\theta$ is a periodic point we redefine $\Omega$ to include only the distinct (nonrepeating) preimages of $\theta$. In this way, $\Omega$ is finite and distinct when $\theta$ is periodic.

The importance of the set $\Omega$ is that it now characterizes the invariant set of $F$.

LEMMA 3.6. If $\Omega$ is finite, then $F$ has a periodic solution (and rational rotation number).

Proof. Suppose $\theta$ is not a periodic point. Let $P_{0}=\min _{k}\left\{\phi_{k}\right\}, P_{1}=\max _{k}\left\{\phi_{k}\right.$ : $\left.\phi_{k}<F(1)\right\}$. Then $P_{0}>0, P_{1}<F(1)$ and $F$ and all its iterates are continuous on $\left[0, P_{0}\right)$ and $\left(P_{1}, F(1)\right]$. There are integers $j_{0}$ and $j_{1}$ such that $F^{\left(j_{0}\right)}\left(P_{0}\right)=\theta, F^{\left(j_{1}\right)}\left(P_{1}\right)$ $=\theta$ so that

$$
F^{\left(j_{0}+2\right)}\left(\left(0, P_{0}\right)\right) \subset\left(P_{1}, F(1)\right), \quad F^{\left(j_{1}+1\right)}\left(\left(P_{1}, F(1)\right)\right) \subset\left(0, P_{0}\right) .
$$


These inclusions follow from the observation that if $F^{\left(j_{0}+2\right)}(0)<P_{1}$, there would be a point of $\Omega$ in $\left(0, P_{0}\right)$ which by construction cannot occur. Similarly $F^{\left(j_{1}+2\right)}(1)<$ $P_{0}$. Combining these inclusions we find that for $j=j_{0}+j_{1}+3$

$$
F^{(j)}\left(\left(0, P_{0}\right)\right) \subset\left(0, P_{0}\right) \text {. }
$$

A stronger statement is necessary to prove the existence of a fixed point of $F^{(j)}$. It follows from the above arguments that $F^{(j)}(0)>0$ and $\lim _{x \uparrow P_{0}} F^{(j)}(x)<P_{0}$. Thus, by continuity, there is a fixed point of $F^{(j)}$ in $\left(0, P_{0}\right)$ which is a periodic point of period $j$ for $F$.

LEMMA 3.7. If the set $\Omega$ is infinite, then the invariant set of $F$ contains $\Omega$ and is infinite.

Proof. Let $g=\left\{x: x=F^{(k)}(\theta), k=\ldots,-2,-1,0,1,2, \ldots\right\}$. Then $g$ is infinite and $\Omega \subset \mathscr{G}$. Furthermore $F(\mathscr{G})=\mathscr{G}$, so $\mathscr{G}$ is invariant.

We will show later that the invariant set is actually a Cantor set. For now we need to determine the conditions under which the set $\Omega$ is finite or infinite. To explore this question we suppose that $F$ depends smoothly on some parameter $\lambda$, and that for each value of $\lambda$ in some interval $\Lambda$ the function $F(x ; \lambda)$ satisfies the conditions F-I, II, III, IV set forth earlier. In addition we make the following specific assumptions about the dependence of $F$ on $\lambda$.

$(\lambda-\mathrm{I})$ For $\lambda \in \Lambda, d \theta / d \lambda$ and $\partial F(x ; \lambda) / \partial \lambda<0, x \in[0,1], x \neq \theta$. Clearly $\rho$ is a nonincreasing function of $\lambda$ when $(\lambda-I)$ holds.

To see the implications of this parametric dependence prescribed by $(\lambda-I)$ we make a few preliminary calculations. Suppose $\phi(\lambda)$ is a point for which $F^{(n)}(\phi(\lambda) ; \lambda)$ $=\theta$ then

$$
\frac{d \phi}{d \lambda}=-\left.\sum_{i=1}^{n-1} \frac{F_{\lambda}\left(F^{(i)}(x ; \lambda) ; \lambda\right)}{\Pi_{k=0}^{i} F_{x}\left(F^{(k)}(x ; \lambda) ; \lambda\right)}\right|_{x=\phi(\lambda)}+\left.\frac{d \theta / d \lambda}{\prod_{k=0}^{n-1} F_{x}\left(F^{(k)}(x ; \lambda) ; \lambda\right)}\right|_{x=\phi(\lambda)} .
$$

This calculation follows from the fact that

$$
\frac{\partial F^{(n)}(x ; \lambda)}{\partial x}=\prod_{k=0}^{n-1} F_{x}\left(F^{(k)}(x ; \lambda) ; \lambda\right)
$$

and

$$
\frac{\partial F^{(n)}(x ; \lambda)}{\partial \lambda}=\sum_{i=1}^{n-1}\left\{F_{\lambda}\left(F^{(i)}(x ; \lambda) ; \lambda\right) \cdot \prod_{k=i+1}^{n-1} F_{x}\left(F^{(k)}(x ; \lambda) ; \lambda\right)\right\} .
$$

From (3.3) we see that

$$
d c(\lambda) / d \lambda=F_{\lambda}(1 ; \lambda)+F_{x}(1 ; \lambda) F_{\lambda}\left(\theta^{-} ; \lambda\right)
$$

where

$$
c(\lambda)=F(1 ; \lambda) \text { and } F\left(\theta^{-} ; \lambda\right) \equiv \lim _{x \uparrow \theta} F(x ; \lambda) .
$$

Thus we see that all preimages of $\theta$ satisfy $d \phi / d \lambda>0$ while $d c / d \lambda<0$, and $(d / d \lambda) F(0 ; \lambda)<0$. There are at most two values $\lambda \in \Lambda$, say $\lambda_{n}<\lambda_{n+1}$, for which $F^{(k)}\left(0 ; \lambda_{k}\right)=\theta, k=n, n+1$, and there are no values of $\lambda \in\left(\lambda_{n}, \lambda_{n+1}\right)$ for which 
$F^{(k)}(0, \lambda)=\theta, k=n, n+1$. The unique $\lambda$ value at which $\phi_{1}=F(0)$ is the value $\lambda_{n+1}$.

The idea of the discussion which follows is to allow the parameter to vary throughout the interval $\left(\lambda_{n}, \lambda_{n+1}\right)$ and to show that for some values of $\lambda$ the set $\Omega$ is infinite whereas for most values of $\lambda$ the set $\Omega$ terminates. The proof proceeds inductively as we try to determine subintervals of $\left(\lambda_{n}, \lambda_{n+1}\right)$ such that $\phi_{k} \in \Omega$ has a preimage $\phi_{k+1}$ in $(0, F(0)$ ]. To see that some such sequences must terminate we have

LEMMA 3.8. If an element $\phi_{k}$ of $\Omega$ belongs to $[c, F(0)]$, then the sequence $\Omega$ terminates with $\phi_{k}$.

Recall also that the sequence $\Omega$ terminates if $\theta$ is a periodic point, which will be the case when $\phi_{k}=F(0)$ or $F(1)=c$.

The easiest way to visualize how the sequence $\Omega$ terminates is with a simple plot. In Figure 2 we have plotted $\theta, F(0)$ and $F(1)$ as functions of $\lambda$ as well as a few preimages of $\theta$. By plotting a few more preimages of $\theta$ one easily sees that this construction divides intervals into three subintervals and that the sequence $\Omega$ terminates on the middle of these subintervals. This is the construction of a Cantor set. The proof that follows gives the detailed description of this inductive process.

LEMMA 3.9. There are subintervals $\Lambda_{0}$ and $\Gamma_{0}$ of $\left(\lambda_{n}, \lambda_{n+1}\right]$ with $\Lambda_{0}<\Gamma_{0},\left(\lambda_{n}, \lambda_{n+1}\right]$ $=\Lambda_{0} \cup \Gamma_{0}$ such that when $\lambda \in \Lambda_{0}$ then $\phi_{1} \in(0, c)$ and when $\lambda \in \Gamma_{0}, \phi_{1} \in[c, F(0)]$.

Proof. By definition for $\lambda \in\left(\lambda_{n}, \lambda_{n+1}\right), F^{(n)}\left(\phi_{1} ; \lambda\right)=\theta$ and as $\lambda$ changes from $\lambda_{n}$ to $\lambda_{n+1}, \phi_{1}$ moves from 0 to $F(0 ; \lambda)$. By assumption $(\lambda-I)$ there is a unique value $\lambda^{*} \in\left(\lambda_{n}, \lambda_{n+1}\right)$ for which $\phi_{1}\left(\lambda^{*}\right)=F\left(1 ; \lambda^{*}\right) \equiv c\left(\lambda^{*}\right)$. Define $\Lambda_{0}=\left(\lambda_{n}, \lambda^{*}\right)$ and $\Gamma_{0}$ $=\left[\lambda^{*}, \lambda_{n+1}\right]$. When $\lambda \in \Lambda_{0}$ the sequence $\Omega$ can be continued beyond $\phi_{1}$ whereas when $\lambda \in \Gamma_{0}$ the sequence $\Omega$ terminates with $\phi_{1}$.

Lemma 3.10. There are infinite sequences of disjoint subintervals of $\Lambda_{0}$ denoted $\Lambda_{1}$, $\Lambda_{2}, \ldots$ and $\Gamma_{1}, \Gamma_{2}, \ldots$ with $\Lambda_{1}<\Gamma_{1}<\Lambda_{2}<\Gamma_{2}<\ldots$ with

$$
\Lambda_{0}=\bigcup_{i=1}^{\infty}\left(\Lambda_{i} \cup \Gamma_{i}\right)
$$

such that if $\lambda \in \Lambda_{k} \cup \Gamma_{k}$, the set of preimages $\Omega$, has elements $\phi_{1}, \phi_{2}, \ldots, \phi_{k}, \phi_{k+1}$ with $\phi_{1}>\phi_{2}>\cdots>\phi_{k}$. If $\lambda \in \Lambda_{k}$ then $\phi_{k+1} \in\left(\phi_{1}, c\right)$ whereas if $\lambda \in \Gamma_{k}$ then $\phi_{k+1} \in[c, F(0)]$. In the latter case, the sequence $\Omega$ terminates with $\phi_{k+1}$.

Proof. When $\lambda \in \Lambda_{0}, \phi_{1} \in(0, c)$ so that it has a preimage $\phi_{2}$ somewhere in $\left(0, F(0)\right.$ ] whose location changes as $\lambda$ changes. Define $\Lambda_{1}$ to be the subinterval of $\Lambda_{0}$ for which $\phi_{2} \in\left(\phi_{1}, c\right)$ and $\Gamma_{1}$ to be the subinterval of $\Lambda_{0}$ for which $\phi_{2} \in$ [c, $F(0)]$. The intervals $\Lambda_{1}$ and $\Gamma_{1}$ are uniquely defined since by assumption $(\lambda-I)$, $\phi_{2}^{\prime}(\lambda)>c^{\prime}(\lambda)$. Furthermore, the left endpoint of $\Lambda_{1}$ is $\lambda_{n}$ since when $\phi_{1}$ is close to 0 , $\phi_{1}<\phi_{2}$.

Define the intervals $\Lambda_{k}$ and $\Gamma_{k}$ inductively to be the subintervals of $\Lambda_{0}$ such that when $\lambda \in \Lambda_{k} \cup \Gamma_{k}, \phi_{1}$ has preimages $\phi_{k}<\phi_{k-1}<\cdots<\phi_{2}<\phi_{1}$, and such that $\phi_{k+1} \in\left(\phi_{1}, c\right)$ when $\lambda \in \Lambda_{k}$ or $\phi_{k+1} \in[c, F(0)]$ when $\lambda \in \Lambda_{k}$. Notice that the 
preimage of $\phi_{k}$ lies either in $\left(\phi_{1}, F(0)\right]$ or in $\left(0, \phi_{k}\right)$. By virtue of assumption $(\lambda-I)$, these intervals are uniquely defined and cover $\Lambda_{0}$. To see that an infinite number of such intervals exist, let $\phi^{*}$ be the preimage of $\phi_{1}$ for which $F^{(n+1)}\left(\phi^{*}\right)=\phi_{1}$ (even though $\phi^{*}$ may fall out of the domain of definition of $\left.F\right)$. Recall that $F^{(n-1)}\left(\phi_{1}\right)=$ $\theta$ so that $\phi^{*}<\phi_{1}$ and in fact it may be that $\phi^{*}<0$. Now,

$$
\left(1 / t^{n+1}\right)\left|\phi_{1}-c\right| \geqslant\left|\phi_{1}-\phi^{*}\right| \geqslant\left(1 / s^{n+1}\right)\left|\phi_{1}-c\right| \text {. }
$$

Clearly $\phi^{*} \in\left(0, \phi_{1}\right)$ if $\left|\phi_{1}-c\right|$ is small enough. Thus if $\lambda$ is close enough to the right endpoint of $\Lambda_{0}, \phi_{2}<\phi_{1}$. Similarly, if $\phi_{1}$ has preimages $0<\phi_{j}<\phi_{j-1}$ $<\cdots<\phi_{2}<\phi_{1}<c$ then let $\phi^{*}$ be the preimage of $\phi_{j}$ for which $F^{(n+1)}\left(\phi^{*}\right)=\phi_{j}$. Then $\phi^{*}<\phi_{j}$ and

$$
\left(1 / t^{n+1}\right)\left|\phi_{j}-\phi_{j-1}\right| \geqslant\left|\phi^{*}-\phi_{j}\right| \geqslant\left(1 / s^{n+1}\right)\left|\phi_{j}-\phi_{j-1}\right|
$$

so that

$$
\begin{aligned}
\left|\phi^{*}-c\right| & =\left|\phi^{*}-\phi_{j}\right|+\left|\phi_{j}-\phi_{j-1}\right|+\cdots+\left|\phi_{2}-\phi_{1}\right|+\left|\phi_{1}-c\right| \\
& \leqslant \sum_{k=0}^{j} \frac{1}{t^{k(n+1)}}\left|\phi_{1}-c\right|=\left|\phi_{1}-c\right| \frac{1-1 / t^{(j+1)(n+1)}}{1-1 / t} \equiv k \cdot\left|\phi_{1}-c\right| .
\end{aligned}
$$

Certainly, $\phi^{*} \in(0, c)$ provided $\left|\phi_{1}-c\right| \leqslant|c| / k$. In other words, if $\phi_{1}$ is close enough to $c, \phi^{*}=\phi_{j+1}$ is the next preimage of $\phi_{1}$ in $(0, c)$ satisfying $\phi_{j+1}<\phi_{j}$. Of course for a fixed value of $\lambda$ there are only a finite number of ordered preimages of $\phi_{1}$ since

$$
\left|\phi^{*}-c\right| \geqslant\left|\phi_{1}-c\right| \frac{1-1 / s^{(j+1)(n+1)}}{1-1 / s}>|c|
$$

for a fixed $\phi_{1}$ and $j$ large enough.

We observe that as $\lambda$ moves through $\Lambda_{k}$ the preimage $\phi_{k+1}$ moves through $\left(\phi_{1}(\lambda), c(\lambda)\right)$. Simultaneously $\phi_{k+1}$ has preimages in $\Omega, \phi_{k+2}>\phi_{k+3}>\cdots>\phi_{2 k}$ for which $\phi_{k+j} \in\left(\phi_{j}, \phi_{j-1}\right), j=2,3, \ldots, k$. Thus, there is a preimage of $\phi_{k+1}$ in $\Omega$, either $\phi_{2 k+1}$ or $\phi_{2 k+2}$, which is in the interval $\left(\phi_{1}, F(0)\right)$. It is the location of this particular preimage which is most important.

Definition 3.11. Let $\Omega^{\prime}$ be the subset of $\Omega$ which belongs to $\left(\phi_{1}, F(0)\right]$.

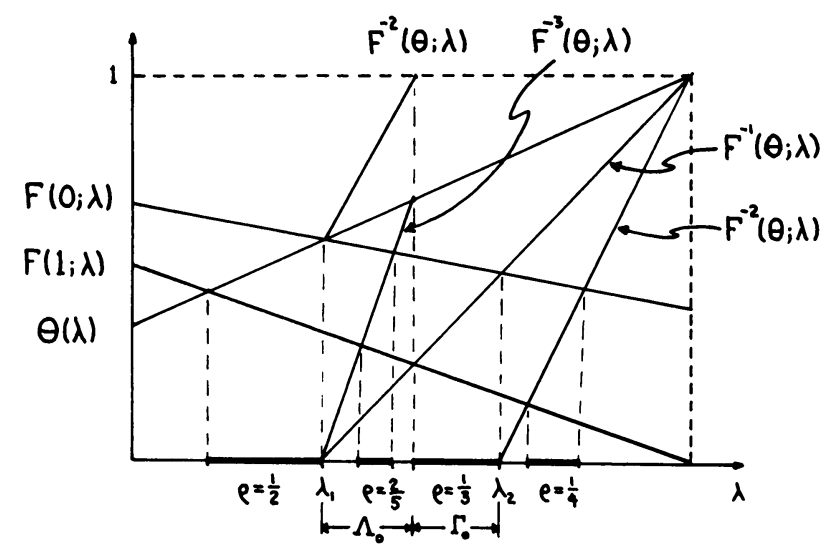

FIGURE 2 
LEMMA 3.12. Let $\phi_{k}$ be an element of $\Omega^{\prime}$ and let $\Delta_{k}$ be the subinterval of $\Lambda_{0}$ for which $\phi_{k}$ is continuously defined, and has a preimage in $\Omega^{\prime}$. Suppose that as $\lambda$ increases through $\Delta_{k}, \phi_{k}$ increases through $\left(\phi_{1}, c\right]$. Then there are three disjoint subintervals of $\Delta_{k}$ denoted $A_{k}, B_{k}, C_{k}$ with $A_{k}<B_{k}<C_{k}$ and $\Delta_{k}=A_{k} \cup B_{k} \cup C_{k}$ such that as $\lambda$ increases through $A_{k}$ or $C_{k}$ the preimage of $\phi_{k}$ in $\Omega^{\prime}$, denoted $\phi_{k+1}$, increases through $\left(\phi_{1}, c(\lambda)\right)$, whereas $\lambda$ increases through $B_{k}, \phi_{k+1}$ increases through $[c(\lambda), F(0)]$.

The interval $B_{k}$ is a subinterval of $\Delta_{k}$ on which the sequence of preimages $\Omega$ (or $\Omega^{\prime}$ ) terminates, whereas on $A_{k}$ or $C_{k}$ the sequence continues and furthermore the properties of $A_{k}$ and $C_{k}$ are the same as $\Delta_{k}$. Thus, Lemma 3.12 constitutes an inductive step.

LEMMA 3.13. The subset of $\Lambda_{k}$ on which $\Omega$ is infinite is uncountable and of zero measure.

Proof. The subset of $\Lambda_{k}$ on which $\Omega$ is infinite is formed inductively using Lemma 3.12 and discarding those intervals $B_{k}$ on which the sequence $\Omega^{\prime}$ terminates. This construction is the same construction used to form a Cantor set (discarding closed rather than open intervals). Thus $\Omega$ is infinite on a set which differs from a Cantor set by only a countably infinite number of endpoints. Furthermore, this set has measure zero since at each step of the construction in going from set $\Lambda_{k}^{i}$ to $\Lambda_{k}^{i+1}$, we know that measure $\left(\Lambda_{k}^{i+1}\right)=\alpha_{i}$ measure $\left(\Lambda_{k}^{i}\right)$, where $\alpha_{i}<1 . \alpha_{i}$ is bounded away from zero for large $i$ since for all $\lambda \in \Lambda_{0}, F(0 ; \lambda)>c(\lambda)$ so that each of the intervals $B_{i}$ must have measure $B_{i} /$ measure $\Delta_{i}>0$ even though measure $B_{i} \rightarrow 0$ for large $i$.

To summarize in concise form what has just been proven we have

THEOREM 3.14. Suppose $F(x ; \lambda)$ satisfies properties F-I, II, III, IV and $(\lambda-I)$ for all $\lambda \in \Omega$. Then, for all but an uncountable set of $\lambda \in \Omega$ of measure zero, the rotation number is rational and all solutions of $x_{n+1}=F\left(x_{n} ; \lambda\right)$ are periodic or eventually periodic.

A similar result holds for 1-1 mappings of the circle onto itself [8]. The rotation number is rational on a dense set of parameter values and is irrational on the complementary set. However, this set has positive measure. Thus, we see that when $F(0)=F(1)$, the set of values $\lambda$ for which the rotation number is irrational has increased in size to a set of positive measure. In fact as $F(0)-F(1)$ decreases it is apparent that the thickness of the Cantor set is increasing and becomes infinite when $F(0)=F(1)$.

There are a number of general features of the solution of (1.1) which can be deduced as a result of Theorem 3.14. First, it should be noted that according to the definition of chaos (Definition 1.1) the motion of (1.1) is never chaotic if F-I, II, III and IV hold, since the rotation number is not dependent on initial data. On the other hand it is possible that the invariant set has a very complicated structure which might be considered chaotic.

COROllary 3.15. If F-I, II, III, IV hold and $\rho$ is irrational then the invariant set of $F, \cap_{i=0}^{\infty} F([0,1])$ is a Cantor set. 
Proof. If the rotation number is irrational, then by the construction of the previous section, no preimage of $\theta$ is in $[F(1), F(0)]$. Thus, the images of $[F(1), F(0)]$ never contain $\boldsymbol{\theta}$.

Now regard the mapping $F$ as a mapping of the circle which is cut at zero, rotated and laid back onto the circle with a gap removed. The gap removed is of course the interval $(F(1), F(0))$. After another iteration, the circle has two gaps removed, specifically $(F(1), F(0))$ and $F((F(1), F(0)))$. As iteration proceeds more gaps are removed from the circle in the same way. Furthermore, the process will continue indefinitely unless one of the previously removed gaps contains $\theta$ at some later iteration. However, this can never happen when $\rho$ is irrational since no image of $(F(1), F(0))$ ever contains $\theta$. When this construction continues indefinitely removing gaps from the circle, it is clear that a Cantor set is being constructed as stated. At the $n$th step the interval $F^{(n)}((F(1), F(0)))$ is removed. There is no guarantee, however, that this Cantor-like set has measure zero. However, if the slope of the function $F$ is uniformly bounded by one, the measure of the invariant set is zero.

Corollary 3.16. Suppose F satisfies F-I, II, III, IV and $s<1$.

(a) If $\rho$ is irrational, the invariant set is uncountable, measure zero (Cantor set).

(b) If $\rho$ is rational, the periodic solution is unique and globally attracting.

According to Theorem 3.14 , the Cantor invariant set is a rarity.

Corollary 3.17. Suppose F satisfies F-I, II, III, IV. If (1.1) has rotation number $\rho$ which is rational and the periodic solution does not include the point $\theta$, it is structurally stable. If $\rho$ is irrational the invariant set is structurally unstable.

Proof. The solution of (1.1) depends continuously on parameters or perturbations provided no point of the solution hits $\theta$. If $F(x)$ has $\rho$ irrational, then so does $f(x ; \lambda)=F(x)+\lambda(x-\theta)^{2}$ for $\lambda=0$. By Theorem 3.14, there is no interval about $\lambda=0$ for $f(x ; \lambda)$ for which the Cantor solution persists.

4. Noninvertible maps. Suppose that the function $F$ satisfies F-I, II, III, and F-V: $F(1)>F(0)$. The function $F$ now maps the circle onto itself but is not 1-1. We assume first that $F(0)=0$, that $F$ is right continuous at $\theta$, that there are no other fixed points of the mapping and that $F(1)<\theta$. The ideas used here will be exploited later for the case $F(0)>0$.

With $F(0)=0$, any point which maps into 0 has rotation number 0 . In particular $\rho(0)=0$. We show first that $\rho(x)$ takes on many other values depending on $x$.

LEMmA 4.1. Let $k>0$ be the smallest integer such that $\theta<F^{(k)}(1)<1$. Then there is an $x \in[0,1]$ with $\rho(x)=1 /(k+1)$.

Proof. Suppose $F^{(k)}(1)>\theta, F^{(j)}(1)<0$ for $j=1,2, \ldots, k-1$. Let $\alpha$ be any point $\alpha<1$ for which $F^{(k)}(\alpha)>\theta$. Then there is an $\varepsilon_{0}>0$ such that for all $0<\varepsilon<\varepsilon_{0}, F(\theta-\varepsilon) \geqslant \alpha$. Define the $k+1$ intervals $A_{0}=[\theta, \alpha], A_{j}=\left[0, F^{(j)}(\alpha)\right]$, $j=1,2, \ldots, k-1, A_{k}=[0, \theta-\varepsilon]$ for $\varepsilon<\varepsilon_{0}$ fixed. Notice that $F$ is continuous on each $A_{j}$, and that $F\left(A_{i}\right) \supset A_{i+1}, i=0,1,2, \ldots, k-1$ and $F\left(A_{k}\right) \supset A_{k} \cup A_{0}$. 
These inclusions allow us to conclude that $F^{(k+1)}\left(A_{0}\right) \supset A_{0}$ so that, by Lemma 2.3, there is a point of period $k+1$ contained in $A_{0}$. For this point $\rho(x)=1 /(k+1)$.

COROLlaRY 4.2. The rotation number $\rho(x)$ takes on all values in the interval $[0,1 /(k+1)]$.

Proof. From the construction of the above proof, we see that we have a shift on the sequence space of interval $A_{0}, A_{1}, \ldots, A_{k}$ with the restriction that admissible sequences consist of strings of $A_{0}, A_{1}, A_{2}, \ldots, A_{k}$ with any number of additional $A_{k}$ between the strings.

To calculate the rotation number for a given sequence, we need to know the number of occurrences of $A_{0}$ per iterate as $n$ approaches $\infty$. This is easily calculated from a given sequence when we realize that a string $A_{0}, A_{1}, \ldots, A_{k}$ contributes $1 /(k+1)$ and each additional $A_{k}$ contributes $0 / 1$ to the rotation number. The rotation number is calculated by taking the ratio of the sums of all numerators and the sums of all denominators.

After $n$ such strings the approximate rotation number can be written as

$$
\rho_{n}=\frac{\Sigma^{n} s_{m}}{n+\Sigma^{n} k s_{m}}, \quad s_{m}=0,1,
$$

the element $\rho_{n}$ can be viewed as an entry in a Pascal's triangle. For example, with $k=2$, a partial display of the possible $\rho_{n}$ is found in Figure 3. It is not hard to see that all the elements of a given row are ordered along the row. Once a rational number is produced in this table it is repeated in lower rows by repeating the strings in the sequence which originally produced it. To see that all rational numbers between 0 and $1 /(k+1)$ are produced and every irrational number is produced in the limit $n \rightarrow \infty$ we look at two neighboring elements of the $n$th row of the triangle. For these two elements, $\rho_{n}^{1}$ and $\rho_{n}^{2}$, say

$$
\begin{aligned}
\rho_{n}^{2}-\rho_{n}^{1} & =\frac{\sum^{n} s_{m}+1}{n+\sum^{n} k s_{m}+k}-\frac{\sum^{n} s_{m}}{n+\sum^{n} k s_{m}} \\
& =\frac{n}{\left(n+\sum^{n} k s_{m}+k\right)\left(n+\sum^{n} s_{m} k\right)}<\frac{1}{n} .
\end{aligned}
$$

The difference between two elements on a given role decreases as $\boldsymbol{n}$ increases. All rationals between 0 and $1 /(k+1)$ are found somewhere in the infinite table, and every irrational number is reached in the limit $n \rightarrow \infty$.

According to Corollary 4.2, chaotic behavior results whenever there is overlap. The strength of the chaos is in some sense measured by the range of the rotation number $\rho(x)$. The number $\rho(x)$ covers at least the interval $[0,1 /(k+1)]$. However, in the proof of this result there were many points in $x \in[0,1]$ which were not included in these considerations. In fact, by choosing the right endpoints of the intervals to be $k+1$-periodic points, we see that none of the points to the right of the largest $(k+1)$-periodic point are needed in the proof. This suggests that the range of $\rho(x)$ is larger than $[0,1 /(k+1)]$. However, it is not hard to see that the range of $\rho(x)$ is bounded. 


$$
\begin{array}{ccccccccc}
n=1 & & & \frac{0}{1} & \frac{1}{3} & & \\
n=2 & & & \frac{0}{1} & \frac{1}{4} & \frac{1}{3} & \\
3 & & \frac{0}{1} & \frac{1}{5} & \frac{2}{7} & \frac{1}{3} & \\
4 & & \frac{0}{1} & \frac{1}{6} & \frac{1}{4} & \frac{3}{10} & \frac{1}{3} \\
5 & \frac{0}{1} & \frac{1}{7} & \frac{1}{5} & \frac{2}{7} & \frac{4}{13} & \frac{1}{3}
\end{array}
$$

\section{FiguRE 3}

COROllaRy 4.3. $\rho(x)<1 / k$ for all $x \in[0,1]$.

The range of $\rho(x)$ is actually $[0, \beta]$ where $\beta$ is some number $1 /(k+1)<\rho<$ $1 / k$. It is possible to get more accurate estimates of the number $\beta$ by looking more carefully at the image of the point 1 . However, the arguments are quite involved and will not be included in this discussion.

The function $\rho(x)$ is a very complicated function. To get an idea of the shape of this function we have

LEMMA 4.4. If $F^{\prime}(x) \geqslant t>1$ for all $x \neq \theta$, then the function $\rho(x)$ is nowhere continuous on $[0,1]$.

Proof. We show first of all that the points $x \in[0,1]$ for which $\rho(x)=0$ are dense in $[0,1]$. To see this we need only look at all the preimages of $\theta$ in $[0,1]$. It is apparent that the preimages of $\theta$ form an infinite set. In fact, there are points $\theta>\phi_{1}>\phi_{2}>\cdots>\phi_{n}>\cdots$ such that $F^{(j)}\left(\phi_{j}\right)=\theta, j=1,2, \ldots$ For $j>$ $k-1$, each of these points has a preimage in $[\theta, 1]$, which in turn guarantees that between each pair $\left[\phi_{i}, \phi_{i+1}\right]$ there is an infinite set of preimages of $\theta$, specifically the preimages of $\phi_{k-1}, \phi_{k}, \phi_{k+1}, \ldots$, denoted $\phi_{k-1}^{i}, \phi_{k}^{(i)}, \ldots, i=1,2, \ldots$

Now, for $i \geqslant k-1$, the doubly infinite set of preimages again has preimages in $[\theta, 1]$ which again guarantees more preimages between $\left[\phi_{i}, \phi_{i+1}\right]$. Inductively it is clear that there is a cascading infinity of preimages of $\theta$. Furthermore, since $F^{\prime}(x)>1$, the maximal distance between preimages goes to zero as the induction proceeds.

Between any two preimages of $\theta$, there is a point with $\rho(x)=1 /(k+1)$. In fact, a point of period $k+1$ lies between $\left[\phi_{1}, \phi_{i+1}\right]$ for $i=1,2, \ldots, k-1$. Furthermore, preimages of such a point lie in $\left[\phi_{j}, \phi_{j+1}\right]$ for all $j=k, k+1, \ldots$ Thus, by induction, such a preimage lies between any two preimages of $\boldsymbol{\theta}$.

Finally, let $\rho(x)=\alpha$ for some $x \in(0,1)$. Then in any $\varepsilon>0$ neighborhood of $x$, there is a point $x_{0}$ with $\rho\left(x_{0}\right)=0$ and a point $x_{1}$ with $\rho\left(x_{1}\right)=1 /(k+1)$. Clearly $\rho(x)$ is not continuous at $x$.

The requirement $t>1$ is a necessary condition for this result to hold. In fact, it 
is easy to construct examples for which $\rho(x)$ is continuous on intervals when $F^{\prime}(x)$ fails to be everywhere greater than one. One such example can be constructed as follows. Start with a piecewise linear function with positive slope less than one which has a period-two solution. This period-two solution is obviously globally stable since $F$ is in the class of functions discussed in \$3. Denote the period-two solution by $x_{1}, x_{2}$ where $x_{2}>x_{1}$. Then for two points $y_{1}<x_{1}$ and $y_{2}>x_{2}$ change the function $F$ on the domain $0 \leqslant x \leqslant y_{1}$ and $y_{2}<x \leqslant 1$ so that $F(0)<F(1)$ and so that $F$ remains smooth for $x \neq \theta$. Clearly there still remains a period-two solution which is at least locally stable and $\rho(x)=\frac{1}{2}$ on some subinterval of $[0,1]$, even though $\rho(x)$ covers an interval containing $\frac{1}{2}$.

This example leads us to another observation about the structure of $\rho(x)$.

Lemma 4.5. Suppose $\rho(x)=\alpha$ on some subinterval of $[0,1]$. Then $\rho(x) \neq \alpha$ on at most a Cantor set in $[0,1]$.

Proof. If $\rho(x) \neq \alpha$ then all images of $x$ must avoid the interval, say $A_{0}$ on which $\rho(x)=\alpha$. Without loss of generality $A_{0} \subset[\phi, \theta]$. To see what points can have $\rho(x) \neq \alpha$, start with the interval $[\theta, 1]$ and look at the image of this interval. At each iteration, discard any subinterval of $[\theta, 1]$ whose image intersects $A_{0}$. It is not hard to see that this removal process must occur infinitely often, leaving at most a Cantor set in $[\theta, 1]$ which has $\rho(x) \neq \alpha$. It is not guaranteed, however, that this Cantor set has measure zero.

The structure of $\rho(x)$, although somewhat complicated, must belong to one of the classes described in Lemma 4.4 or 4.5

LEMMA 4.6. Either $\rho(x)$ is constant on all but a Cantor set, or $\rho(x)$ is nowhere continuous.

Proof. Suppose $\rho(x)$ is nonconstant on some interval about $x_{0}$. For any $\delta>0$, the $\delta$-neighborhood of $x_{0}$ must contain at least two points $a<x_{0}$ and $b>x_{0}$ for which $\rho(a) \neq \rho\left(x_{0}\right) \neq \rho(b)$. Since $\rho(a) \neq \rho\left(x_{0}\right)$ there must be a point $\left[a, x_{0}\right]$ which is a preimage of $\theta$. Similarly there is a preimage of $\theta$ in $\left[x_{0}, b\right]$. But as noted in the proof of Lemma 4.4 , there is a point with $\rho=1 /(k+1)$ between every pair of preimages of $\theta$. Thus, $\rho$ takes on the values $1 /(k+1)$ and 0 in any $\delta$-neighborhood of $x_{0}$. Clearly $\rho$ is not continuous at $x_{0}$.

All of the above statements assume that $F(0)=0, F$ is right continuous at $\theta$, $F(1)<\theta$ and there are no other fixed points. It is not difficult to see that the assumptions of right continuity of $F$ and $F(1)<\theta$ had nothing to do with the main results and can be dropped, although if $F(1)>\theta$, a good estimate for the range of $\rho(x)$ is more difficult to obtain.

If $F(0) \neq 0$ but there are other fixed points, then the previous observations carry over if the smallest closed interval containing all fixed points is a proper subset of $[F(0), F(1)]$. The proof follows by looking at the mapping on the restricted interval 
$[a, b]$ where

$$
\begin{aligned}
& a=\max [\{0\},\{x \mid F(x)=x, x<\theta\}], \\
& b=\min [\{1\},\{x \mid F(x)=x, x>\theta\}] .
\end{aligned}
$$

Define $A$ to be the point less than $\theta$ for which $F(A)=a$ and $B$ to be the point greater than $\theta$ for which $F(B)=b$. Then notice that all points in the interval $(A, \theta)$ have $\rho(x)=1$ and all points in $(\theta, B)$ have $\rho(x)=0$ since they eventually go to a fixed point. By defining $F(x)=F(b)$ for $x \in(A, \theta)$ and $F(x)=F(a)$ for $x \in$ $(\theta, B)$, we have a new mapping with the same features as above and $\rho(x)$ unchanged.

The situation for $F(0) \neq 0$ and no other fixed points is not completely resolved. It is apparent that chaos results if $[F(0), F(1)]$ is "sufficiently large" so as to allow periodic solutions of different period. However what is meant by "sufficiently large" is not precisely known. In summary, we have the following general result.

THEOREM 4.7. Suppose F satisfies F-I, II, III and IV. Let I be the smallest closed interval containing all fixed points of $F$.

(i) If $I$ is nonempty, then $\rho(x)$ covers a nontrivial interval $[\alpha, \beta], \alpha \neq \beta$ if and only if either $I$ is a proper subset of $[F(0), F(1)]$ or $I=[0,1]$. If $I \cap[0, \theta) \neq \varnothing$ then $\alpha=0$ and if $I \cap[\theta, 1] \neq \varnothing$ then $\beta=1$. Furthermore, $\rho(x) \neq 0,1$ on $a$ Cantor set. If $F^{\prime}(x)>1$ on $[a, A] \cup[B, b]$, the measure of the Cantor set is zero.

(ii)(a) If $I$ is empty and $[F(0), F(1)]$ is sufficiently large so as to allow at least two periodic solutions with different periods, then $\rho(x)$ covers $[\alpha, \beta], \alpha \neq \beta$.

(b) If $I$ is empty and $F^{\prime}(x)>1$ for $x \in[0,1]$, then $\rho(x)$ covers an interval $[\alpha, \beta]$, $\alpha \neq \beta$ and $\rho(x)$ is a nowhere continuous function of $x$.

It is possible that even though $\rho(x)$ is nowhere continuous, it is a constant $\rho_{0}$ almost everywhere. In fact this is the case if there is an invariant measure for $F$. It is also apparent that the range $[\alpha, \beta]$ of $\rho(x)$ somehow increases with increasing overlap. However, reasonable estimates for $\alpha$ and $\beta$ are difficult to calculate. These and other questions about the structure of $\rho(x)$ are yet unresolved.

\section{REFERENCES}

1. A. N. Sarkovskii, Coexistence of cycles of a continuous map of the line into itself, Ukrain. Mat. $\check{Z} .16$ (1964), 61-71. MR 28 \#3121.

2. T. Y. Li and J. A. Yorke, Period three implies chaos, Amer. Math. Monthly 82 (1975), 985.

3. F. C. Hoppensteadt, J. P. Keener and J. Rinzel, Integrate-and-fire models of nerve membrane response to oscillatory input (to appear).

4. A. Rescigno, R. B. Stein, R. L. Purple and R. E. Poppele, A neuronal model for the discharge patterns produced by cyclic inputs, Bull. Math. Biophys. 32 (1970), 337-353.

5. L. Glass and M. C. Mackey, A simple model for phase locking of biological oscillators, J. Math. Biol. 7 (1979), 339-352.

6. A. Denjoy, Sur les courbes définies par les équations différentielles à la surface du tore, J. Math. Pures Appl. (9) 11 (1932), 333-375.

7. E. A. Coddington and N. Levinson, Theory of ordinary differential equations, McGraw-Hill, New York, 1955. 
8. M. R. Herman, Mesure de Lebesgue et nombre de rotation, Geometry and Topology, Lecture Notes in Math., Vol. 597, Eds., J. Palis and M. de Carmo, Springer, Berlin and New York, 1977, pp. 271-293.

9. J. K. Moser, Stable and random motions in dynamical systems, Ann. of Math. Studies, Princeton Univ. Press, Princeton, N. J., 1973.

10. S. Smale and R. F. Williams, The qualitative analysis of a difference equation of population growth, J. Math. Biol. 3 (1976), 1-4.

11. D. Ruelle, Sensitive dependence on initial condition and turbulent behavior of dynamical systems, Conf. Bifurcation Theory and its Appl., New York Acad. Sci., New York, 1977.

Department of Mathematics, University of Utah, Salt Lake Crty, Utah 84112 (Current address)

Department of Mathematics and Program in Applied Mathematics, University of Arizona, Tucson, Arizona 85721 